\title{
Erythropoietin alleviates post-resuscitation myocardial dysfunction in rats potentially through increasing the expression of angiotensin II receptor type $\mathbf{2}$ in myocardial tissues
}

\author{
HOURONG ZHOU ${ }^{1}$, JIA HUANG ${ }^{2}$, LI ZHU ${ }^{3}$ and $\mathrm{YU} \mathrm{CAO}^{1}$ \\ ${ }^{1}$ Department of Emergency Medicine, West China Hospital, Sichuan University, Chengdu, Sichuan 610041; \\ ${ }^{2}$ Emergency Department, Guizhou Provincial People's Hospital; ${ }^{3}$ Institute of Anesthesia, \\ Guizhou Medical University, Guiyang, Guizhou 550002, P.R. China
}

Received March 13, 2017; Accepted December 1, 2017

DOI: $10.3892 / \mathrm{mmr} .2018 .8473$

\begin{abstract}
Activation of renin-angiotensin system (RAS) is one of the pathological mechanisms associated with myocardial ischemia-reperfusion injury following resuscitation. The present study aimed to determine whether erythropoietin (EPO) improves post-resuscitation myocardial dysfunction and how it affects the renin-angiotensin system. Sprague-Dawley rats were randomly divided into sham, vehicle, epinephrine (EP), EPO and EP + EPO groups. Excluding the sham group, all groups underwent cardiopulmonary resuscitation (CPR) 4 min after asphyxia-induced cardiac arrest (CA). EP and/or EPO was administrated by intravenous injection when CPR began. The results demonstrated that the vehicle group exhibited lower mean arterial pressure, left ventricular systolic pressure, maximal ascending rate of left ventricular pressure during left ventricular isovolumic contraction and maximal descending rate of left ventricular pressure during left ventricular isovolumic relaxation $(+\mathrm{LVdP} / \mathrm{dt}$ max and $-\mathrm{LVdP} / \mathrm{dt}$ max, respectively), and higher left ventricular end-diastolic pressure, compared with the sham group following return of spontaneous circulation (ROSC). Few significant differences were observed concerning the myocardial function between the vehicle and EP groups; however, compared with the vehicle group, EPO reversed myocardial function indices following ROSC, excluding-LVdP/dt max. Serum renin and angiotensin (Ang) II levels were measured by ELISA. The serum levels of renin and Ang II were significantly increased in the vehicle group compared with the sham group, which was also observed for the myocardial expression of renin and Ang II receptor
\end{abstract}

Correspondence to: Professor Yu Cao, Department of Emergency Medicine, West China Hospital, Sichuan University, 37 Guo Xue Xiang, Chengdu, Sichuan 610041, P.R. China

E-mail: dryu.cao@gmail.com

Key words: cardiac arrest, post-resuscitation myocardial dysfunction, erythropoietin, renin, angiotensin II receptor type 2, epinephrine type 1 (AT1R), as determined by reverse transcription-quantitative polymerase chain reaction and western blotting. EPO alone did not significantly reduce the high serum levels of renin and Ang II post-resuscitation, but changed the protein levels of renin and AT1R expression in myocardial tissues. However, EPO enhanced the myocardial expression of Ang II receptor type 2 (AT2R) following ROSC. In conclusion, the present study confirmed that $\mathrm{CA}$ resuscitation activated the renin-Ang II-AT1R signaling pathway, which may contribute to myocardial dysfunction in rats. The present study confirmed that EPO treatment is beneficial for protecting cardiac function post-resuscitation, and the roles of EPO in alleviating post-resuscitation myocardial dysfunction may potentially be associated with enhanced myocardial expression of AT2R.

\section{Introduction}

Post-resuscitation myocardial dysfunction is an important morbidity of post-resuscitation syndrome. The mortality rate following cardiopulmonary resuscitation (CPR) in out-of-hospital cardiac arrest (CA) is $\sim 75 \%$, one-third of which is thought to be caused by myocardial dysfunction (1). Post-resuscitation myocardial dysfunction always occurs following return of spontaneous circulation (ROSC) and has long-term effects. Left ventricular ejection fractions prior to and following CA are associated with the prognosis of CA (2). In addition, one study reported a potentially harmful effect of epinephrine (EP) on post-ROSC hemodynamics, with a lower cardiac index in patients that received higher cumulative EP doses during CPR (3).

Ischemia-reperfusion injury is one of the pathological mechanisms associated with myocardial dysfunction following resuscitation (2). This injury leads to the activation of the systemic and local renin-angiotensin (Ang) system (RAS), subsequently leading to increased serum levels of renin and Ang II, and activation of Ang II receptors (4). Furthermore, EP stimulates the $\beta 1$ receptor of glomerular parietal cells, promotes renin secretion and induces the upregulation of serum renin and Ang II levels (5). The binding of Ang II to Ang II receptor type 1 (AT1R) causes myocardial cell damage and heart failure (6). However, Ang II receptor type 2 (AT2R) 
counteracts the canonical signaling of RAS that is mediated by AT1R. It has been previously demonstrated that AT2R inhibits ligand-induced AT1R signaling in a protein kinase C-dependent manner (7), and the AT2R agonist C21 significantly improves post-myocardial infarct cardiac function (8).

Erythropoietin (EPO) has been previously reported to protect cardiac function and lessen post-resuscitation myocardial dysfunction (5,9-11). Specifically, 5,000 U/kg EPO reduced the area of myocardial necrosis and restored cardiac function to an almost normal level within a week (11). In addition, 1,200 U/kg EPO reduced post-resuscitation myocardial stunning of ventricular fibrillation in pig models of ROSC (9). Similar results were obtained in rats models of asphyxia-induced CA (5) and hemorrhagic shock (10). Furthermore, additional studies have indicated that EPO treatment reduces the activity of the RAS (12-14), and also reduces inflammation and oxidative stress (12).

Based on the established roles of the RAS in myocardial ischemia-reperfusion injury following CA, it may be hypothesized that EPO may alleviate post-resuscitation myocardial dysfunction by regulating the RAS, which, to the best of our knowledge, has not been previously investigated. Therefore, the present study aimed to elucidate whether EPO improves post-resuscitation myocardial dysfunction and how it affects the RAS. The cardiac function, serum renin and Ang II levels, and the myocardial expression of renin, AT1R and AT2R, in rats following CA resuscitation with or without EPO pretreatment (administered when CPR began) were compared. The effect of EP addition on CA resuscitation was also observed.

\section{Materials and methods}

Experimental design and grouping. The present study was approved by the Animal Ethics Committee of Guizhou Provincial People's Hospital (approval no. 2012026; Guiyang, China) and performed in accordance with the International Ethical Guidelines for Animal Research (15). Male ( $n=75)$ and female $(n=75)$ Sprague-Dawley rats (8 weeks old) with a body weight of 350-450 g were provided by the Experimental Animal Center of Guiyang College of Traditional Chinese Medicine (License no. SCXK Chongqing 2012-0005; Guiyang, China). Animals were housed at a constant temperature of $22^{\circ} \mathrm{C}$, humidity of $50 \%$, and $\mathrm{CO}_{2}$ concentration of $0.04 \%$. Rats received food and water ad libidum the night before the experiment, but were fasted and water-deprived during the experiments. Light was kept constant during the experiment.

Sprague-Dawley rats were randomly divided into the following five groups: Sham-operated group (sham group, $\mathrm{n}=30$ ); CA resuscitation group (vehicle group, $\mathrm{n}=30$ ); CA resuscitation + EP group (EP group, $n=30)$; CA resuscitation + EPO group (EPO group, $n=30$ ); and $\mathrm{CA}$ resuscitation $+\mathrm{EP}+\mathrm{EPO}$ group $(\mathrm{EP}+\mathrm{EPO}$ group, $\mathrm{n}=30)$. The process of $\mathrm{CA}$ resuscitation included CA, CPR and ROSC. A diagram indicating the process is presented in Fig. 1. Electrocardiograms were obtained at baseline (prior to surgery) and at $0,1,2,4$ and $6 \mathrm{~h}$ after ROSC ( $\mathrm{n}=6$ per group for each time-point; however, the same batch of animals were used for electrocardiogram measurements at 0 and $1 \mathrm{~h}$ after ROSC). Samples of blood and cardiac tissues were obtained from each group at baseline and at 2, 4 and $6 \mathrm{~h}$ after $\operatorname{ROSC}$ ( $\mathrm{n}=6$ per group for each time-point).
$C A$ resuscitation and cardiac function monitoring. The night before the operation, the rats were fasted, except for water, and intraperitoneally injected with $45 \mathrm{mg} / \mathrm{kg}$ chloral hydrate for anesthesia, $10 \mathrm{mg} / \mathrm{kg}$ of which was administered every hour to maintain its effect.

Initially, low-volume (or lung protective) mechanical ventilation was performed for $30 \mathrm{~min}$ (i.e. baseline, prior to surgery) to ensure hemodynamic stability in all five groups and to avoid lung injury (16). Animals with a mean arterial pressure (MAP) $<80 \mathrm{mmHg}$, those with excessive surgical bleeding or those with a surgical time $>40$ min were excluded.

CA was caused by asphyxiation, which was induced by turning off the ventilator and by clamping the endotracheal tube. Bradycardia, hypotension and cardiac failure with an MAP $<10 \mathrm{mmHg}$ that occurred shortly after asphyxiation were defined as CA (17). At 4 min after CA, ventilation was restored when chest compression was performed using a Modified Brunswick Animal Heart-Lung Resuscitator (Landswick medical technology, Co. Ltd., Guangzhou, China). The chest compression rate was 200/min with a depth half the chest anteroposterior diameter; the pressing and relaxation times were similar. Chest compression was adjusted to the coronary perfusion pressure, which is $>30 \mathrm{mmHg}$. ROSC was characterized by a continuous MAP of $60 \mathrm{mmHg}$ (17). Resuscitation was terminated if ROSC did not appear after 6 min of continuous chest compressions. The sham operation group underwent: anesthesia, endotracheal intubation and mechanical ventilation. During the whole operation, an incandescent lamp was employed to maintain the rectal temperature at $36.5-37.5^{\circ} \mathrm{C}$. A 14 -gauge cannula was used for percutaneous tracheal intubation under a mechanical ventilation of $80 \mathrm{breaths} / \mathrm{min}$, a tidal volume of $0.65 \mathrm{ml} / 100 \mathrm{~g}$ and a fractional inspired oxygen of $100 \%$ (ALC-V9 Animal Ventilator; Alcott Biotech Co., Ltd., Shanghai, China). A PE-50 tube filled with normal saline was inserted into the right femoral artery to measure the arterial blood pressure, and another PE-50 tube filled with normal saline was inserted into the left ventricle through the right common carotid artery for confirmation of left ventricular pressure waveform. Another saline-filled PE-50 tube was inserted into the right internal jugular vein for fluid infusion. The central venous pressure was recorded. An BL-420S biological data acquisition and analysis system (Techman Software Co., Ltd., Chengdu, China; en.tme.com. $\mathrm{cn} /$ products_detail/product $\mathrm{Id}=45 \mathrm{html}$ ) was connected to monitor electrocardiograms, monitoring the heart rate (HR), MAP, left ventricular systolic pressure (LVSP), left ventricular end-diastolic pressure (LVEDP), maximal ascending rate of left ventricular pressure during left ventricular isovolumic contraction $(+\mathrm{LVdP} / \mathrm{dt} \max$, which represents the left ventricular systolic function) and maximal descending rate of left ventricular pressure during left ventricular isovolumic relaxation $(-\mathrm{LVdP} / \mathrm{dt}$ max, which represents the left ventricular diastolic function).

Drug administration. The drugs were administrated when CPR began. Following $\mathrm{CA}$, the vehicle and sham groups received intravenous injection of $2 \mathrm{ml} / \mathrm{kg}$ normal saline as a control, the EP group was intravenously injected with $0.02 \mathrm{mg} / \mathrm{kg}$ (dosing volume, $2 \mathrm{ml} / \mathrm{kg}$ ) EP (Fuzhou Neptunus Fuyao Pharmaceutical Co., Ltd., Fuzhou, China), the EPO group was intravenously 
injected with 5,000 U/kg recombinant human (rh)EPO (5) (dosing volume, $2 \mathrm{ml} / \mathrm{kg}$; Shandong Ahua Biochemical Co., Ltd., Liaocheng, China) and the EP + EPO group was intravenously injected with 5,000 U/kg rhEPO (dosing volume, $1 \mathrm{ml} / \mathrm{kg}$ ) + $0.02 \mathrm{mg} / \mathrm{kg}$ EP (dosing volume, $1 \mathrm{ml} / \mathrm{kg}$ ).

The randomization code for drug information was delivered in a sealed envelope to the operator. The assessors were blind to grouping and drug information. Experimental failure was reported to the investigator, who then broke the code to determine which group the animal belonged to.

Sampling of blood and cardiac tissues. Blood samples $(6 \mathrm{ml})$ were extracted from the jugular vein and centrifuged at $3,000 \mathrm{x} \mathrm{g}$, for $10 \mathrm{~min}$ at $4^{\circ} \mathrm{C}$ to obtain the supernatant serum. The rats were sacrificed by an intravenous injection of a lethal dose $(250 \mathrm{mg} / \mathrm{kg})$ of pentobarbital. The chest was opened and the heart was isolated from the aortic root, rinsed with phosphate-buffered saline (PBS), and the left and right ventricles were separated. All samples were immediately stored at $-80^{\circ} \mathrm{C}$ in a liquid nitrogen tank.

ELISA for serum renin and Ang II levels. ELISA kits (cat. no. csb-e08702r) were used to measure the serum levels of renin (CUSABIO Biotechnology Co., Ltd., Wuhan, China; sensitivity, $1.17 \mathrm{pg} / \mathrm{ml}$ ) and Ang II (cat. no. csb-e0449r; CUSABIO Biotechnology Co., Ltd.; sensitivity, $3.9 \mathrm{mU} / \mathrm{ml}$ ) in rats. The reagents were placed at room temperature $\left(20-25^{\circ} \mathrm{C}\right)$ for 15-20 min prior to use, and the procedures were performed in strict accordance with the manufacturer's protocol. To minimize errors, the samples were tested twice to obtain the mean average.

Reverse transcription-quantitative polymerase chain reaction (RT-qPCR) detection of myocardial renin, ATIR and AT2R $m R N A$ levels. Myocardial tissues were obtained and TRIzol (Tiangen Biotech Co., Ltd., Beijing, China) was used to extract the total RNA, according to the manufacturer's protocol. Gel electrophoresis was performed to detect the integrity of the total RNA extracted. PrimeScript RT reagent kit with gDNA Eraser (Takara Bio, Inc., Otsu, Japan) was used for RT to produce cDNA at $42^{\circ} \mathrm{C}$ for $50 \mathrm{~min}$ and at $85^{\circ} \mathrm{C}$ for $5 \mathrm{~min}$, according to the manufacturer's protocol. SYBR Premix Ex Taq II (Tli RNase H Plus) and ROX plus (Takara Bio, Inc.) were used for amplification of cDNA with the following program: $95^{\circ} \mathrm{C}$ for $30 \mathrm{sec}$ followed by 45 cycles of $95^{\circ} \mathrm{C}$ for $5 \mathrm{sec}$ and $60^{\circ} \mathrm{C}$ for $40 \mathrm{sec}$. An ABI 7500 Real-Time PCR system (Applied Biosystems; Thermo Fisher Scientific, Inc., Waltham, MA, USA) was used for qPCR. Primers used for qPCR are presented in Table I and the $2^{-\Delta \Delta C q}$ method (18) was used to analyze quantification data and normalize the myocardial mRNA expression levels of renin, AT1R and AT2R to GAPDH mRNA expression. This experiment was performed three times.

Western blot analysis of ATIR and AT2R in the myocardium. Briefly, myocardial tissue was washed three times with PBS buffer and lysed in radioimmunoprecipitation assay lysis buffer (Beyotime Institute of Biotechnology, Haimen, China) on ice. Total protein $(50 \mu \mathrm{g} / \mathrm{sample})$, which was determined using a BCA assay (Sigma-Aldrich; Merck KGaA, Darmstadt,

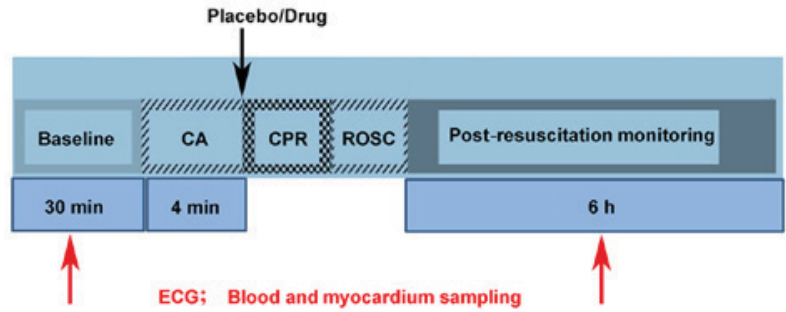

Figure 1. Diagram of the experimental protocol. The process of CA resuscitation included CA, CPR and ROSC. A post-resuscitation monitoring period of $6 \mathrm{~h}$ was employed following ROSC. CA, cardiac arrest; CPR, cardiopulmonary resuscitation; ROSC, return of spontaneous circulation.

Germany), was extracted and separated by $10 \%$ SDS-PAGE and transferred onto polyvinylidene difluoride membranes (EMD Millipore, Billerica, MA, USA). The membranes were blocked with 5\% non-fat milk in TBS-Tween-20 $(10 \mathrm{mM}$ Tris, $\mathrm{pH} 7.4,150 \mathrm{mM} \mathrm{NaCl}$ and $0.1 \%$ Tween-20) at room temperature for $1 \mathrm{~h}$. The primary antibodies against AT1R (cat. no. ab18801; 1:500), AT2R (cat. no. ab92445; 1:500) and GAPDH (cat. no. ab181602; 1:5,000), and horseradish peroxidase-conjugated anti-rabbit (cat. no. cw0234; 1:200; www. cwbiotech.com.) and anti-rat (cat. no. cw0108; 1:200; www. cwbiotech.com) secondary antibodies, were added into a $4 \mathrm{ml}$ centrifuge tube. The sealed membrane was transferred into the box filled with the antibody mixture, incubated overnight at $4^{\circ} \mathrm{C}$ and washed three times with a tris Buffered saline with Tween-20 washing solution. For enhanced chemiluminescence (ECL Western Blotting Substrate; cat. no. 32019; Thermo Fisher Scientific, Inc.) and exposure development, the reaction time was $5 \mathrm{~min}$, and exposure time was $10 \mathrm{~min}$ for AT1R, $1 \mathrm{~min}$ for AT2R and $5 \mathrm{~min}$ for GAPDH. BandScan 4.3 software was used to scan and determine the gray value of the target protein (Gel-Pro analyzer 4.0 (Supplier: Media Cybernetics, USA). The expression of each protein was compared with that of GAPDH and the control group was compared with the other groups.

Statistical analysis. Data were analyzed using SPSS 17.0 (SPSS, Inc., Chicago, IL, USA). Data are presented as the mean \pm standard deviation. Comparisons among multiple subgroups were performed using two-way analysis of variance, followed by Student-Newman-Keuls test. $\mathrm{P}<0.05$ was considered to indicate a statistically significant difference.

\section{Results}

Operation parameters among the groups. No significant differences were observed among the groups $(\mathrm{P}>0.05)$ in terms of weight, chloral hydrate and EP usage, asphyxia time, CPR time, ROSC time and resuscitation success rate (Table II). The overall rate of successful resuscitation from $\mathrm{CA}$ in the present study was $51 \%(120 / 236)$.

EPO alleviates post-resuscitation myocardial dysfunction. Alterations in cardiac function are presented in Table III. No differences were observed in the HR among the five groups. Compared with the sham group, the vehicle group exhibited a lower MAP,LVSP, +LVdP/dt max and -LVdP/dt max, and higher 
Table I. Primers for reverse transcription-quantitative polymerase chain reaction analysis of AT1R, AT2R, renin and GAPDH mRNA expression in rats.

\begin{tabular}{llll}
\hline & \multicolumn{2}{c}{ Primer sequence } & \multicolumn{1}{c}{$\begin{array}{c}\text { Fragment } \\
\text { length, bp }\end{array}$} \\
\cline { 2 - 4 } Gene & \multicolumn{2}{c}{ Forward } & 5-GATGATGCAGGTGACTTTGG-3 \\
AT1R & 5-GTGTTCCTGCTCACGTGTCT-3 & 5-TGGCTAGGCTGATTACATGC-3 & 108 \\
AT2R & 5-GAAGCTCCGCAGTGTGTTTA-3 & 147 \\
Renin & 5-CTGGGAGGCAGTGACCCTCAACATTACCAG-3 & 5-GAGAGCCAGTATGCACAGGTCATCG & 372 \\
GAPDH & 5-ACAACTTTGGCATTGTGGAA-3 & 5-GATGCAGGGATGATGTTCTG-3 & 133 \\
\hline
\end{tabular}

AT1R, angiotensin II receptor type 1; AT2R, angiotensin II receptor type 2.

Table II. Comparison of experimental parameters in each group.

\begin{tabular}{|c|c|c|c|c|c|c|}
\hline \multirow[b]{2}{*}{ Parameter } & \multicolumn{6}{|c|}{ Group } \\
\hline & Sham & Vehicle & EP & $\mathrm{EPO}+\mathrm{EP}$ & EPO & P-value \\
\hline Weight, g & $326.33 \pm 10.33$ & $321.23 \pm 8.89$ & $320.07 \pm 8.39$ & $326.2 \pm 11.15$ & $322.63 \pm 9.80$ & 0.635 \\
\hline Chloral hydrate usage, mg & $122.3 \pm 10.92$ & $121.17 \pm 10.37$ & $120.80 \pm 8.08$ & $124.97 \pm 7.62$ & $121.03 \pm 9.68$ & 0.287 \\
\hline EP usage, $\mu \mathrm{g}$ & NA & NA & $5.55 \pm 0.59$ & $5.90 \pm 0.61$ & NA & 0.442 \\
\hline Asphyxia time, sec & NA & $223.50 \pm 13.60$ & $222.1 \pm 11.00$ & $221.5 \pm 11.5$ & $217.77 \pm 12.52$ & 0.744 \\
\hline CPR time, sec & NA & $296.2 \pm 50.10$ & $294.83 \pm 49.30$ & $269.73 \pm 48.14$ & $290.77 \pm 58.42$ & 0.589 \\
\hline ROSC time, sec & NA & $536.2 \pm 50.10$ & $534.83 \pm 49.31$ & $509.73 \pm 48.14$ & $530.77 \pm 58.42$ & 0.526 \\
\hline $\begin{array}{l}\text { Resuscitation success rate, } \\
\% \text { (proportion) }\end{array}$ & NA & $41.67(30 / 72)$ & $56.60(30 / 53)$ & $58.82(30 / 51)$ & $50.00(30 / 60)$ & 0.498 \\
\hline
\end{tabular}

LVEDP, following ROSC $(\mathrm{P}<0.05$; Table III), indicating that $\mathrm{CA}$ resuscitation led to myocardial dysfunction. Compared with the vehicle group, the only significant differences in the EP group were a higher MAP at $0 \mathrm{~h}$, a higher $+\mathrm{LVdP} / \mathrm{dt}$ $\max$ at 0 and $1 \mathrm{~h}$ after ROSC, and a higher $-\mathrm{LVdP} / \mathrm{dt}$ max at $0 \mathrm{~h}$ after ROSC $(\mathrm{P}<0.05$; Table III); however, $+\mathrm{LVdP} / \mathrm{dt}$ max and $-\mathrm{LVdP} / \mathrm{dt}$ max values remained lower compared with the sham group $(\mathrm{P}<0.05$; Table III). These results indicated that EP addition may not exacerbate the myocardial dysfunction upon CA resuscitation, and it appeared to provide protection, although only at the beginning.

Compared with the vehicle group, the EPO group generally exhibited a higher MAP, LVSP and $+\mathrm{LVdP} / \mathrm{dt}$ max, and lower LVEDP, following ROSC ( $\mathrm{P}<0.05$; Table III), indicating that EPO ameliorated the myocardial dysfunction upon CA resuscitation. However, the long-term effects of EPO were compromised when combined with EP addition; EPO pretreatment almost restored MAP to a normal level at $6 \mathrm{~h}$ after ROSC (P>0.05, EPO group vs. sham group; Table III), but almost no beneficial effect on MAP was observed in the EP + EPO group compared with the EP or vehicle groups between 2 and $6 \mathrm{~h}$ after $\operatorname{ROSC}(\mathrm{P}>0.05$; Table III).
EPO did not reduce the increased serum levels of renin and Ang II post-resuscitation. Serum renin and Ang II levels prior to surgery were similar in all five groups $(\mathrm{P}>0.05$; Tables IV and V, respectively); however, levels were elevated upon CA resuscitation $(\mathrm{P}<0.01$; vehicle group vs. sham group; Tables IV and V). Furthermore, EP addition further augmented the serum renin and Ang II levels $(\mathrm{P}<0.01, \mathrm{EP}$ group vs. vehicle group; Tables IV and V, respectively). EPO treatment did not reduce the increased serum levels of renin and Ang II upon CA resuscitation ( $\mathrm{P}>0.05$; EPO group vs. vehicle group; Tables IV and V, respectively), but EPO did prevent further increases in serum renin levels induced by $\mathrm{EP}$ addition $(\mathrm{P}>0.05$; EP + EPO group vs. vehicle group; Table IV).

EPO did not sustain the downregulation of renin and AT1R expression in myocardial tissues post-resuscitation. EPO suppressed the increase of renin mRNA in myocardial tissues until $4 \mathrm{~h}$ after ROSC (both $\mathrm{P}<0.05$, EPO group vs. vehicle group, 2 and $4 \mathrm{~h}$ following ROSC; Fig. 2), but the regulation was not maintained ( $P>0.05$, EPO group vs. vehicle group, $6 \mathrm{~h}$ following ROSC; Fig. 2). Compared with the sham group, the mRNA levels of renin (Fig. 2) and AT1R (Fig. 3A), in addition 
Table III. Alterations in cardiac function indices in each group.

\begin{tabular}{|c|c|c|c|c|c|}
\hline \multirow[b]{2}{*}{ Parameter } & \multicolumn{5}{|c|}{ Group } \\
\hline & Sham & Vehicle & $\mathrm{EP}$ & $\mathrm{EP}+\mathrm{EPO}$ & EPO \\
\hline \multicolumn{6}{|c|}{$\mathrm{HR}$, beats/min } \\
\hline Baseline & $334.67 \pm 34.90$ & $334.00 \pm 38.38$ & $331.17 \pm 31.77$ & $331.83 \pm 33.65$ & $330.67 \pm 33.49$ \\
\hline $0 \mathrm{~h}$ & $330.67 \pm 37.06$ & $348.00 \pm 35.35$ & $416.17 \pm 32.63$ & $420.17 \pm 33.88$ & $369.33 \pm 33.24$ \\
\hline $1 \mathrm{~h}$ & $328.83 \pm 33.13$ & $322.67 \pm 33.76$ & $371.50 \pm 31.44$ & $361.50 \pm 34.50$ & $336.00 \pm 32.15$ \\
\hline $2 \mathrm{~h}$ & $319.50 \pm 32.52$ & $311.17 \pm 42.98$ & $329.83 \pm 35.56$ & $322.00 \pm 35.13$ & $325.33 \pm 29.86$ \\
\hline $4 \mathrm{~h}$ & $318.14 \pm 27.98$ & $320.00 \pm 46.75$ & $325.17 \pm 37.67$ & $323.50 \pm 26.08$ & $324.67 \pm 33.60$ \\
\hline $6 \mathrm{~h}$ & $313.70 \pm 28.53$ & $322.83 \pm 33.70$ & $312.67 \pm 34.48$ & $313.67 \pm 33.73$ & $312.17 \pm 30.84$ \\
\hline \multicolumn{6}{|c|}{ MAP, $\mathrm{mmHg}$} \\
\hline Baseline & $98.17 \pm 8.07$ & $102.33 \pm 8.46$ & $102.17 \pm 6.26$ & $100.50 \pm 8.30$ & $98.50 \pm 5.65$ \\
\hline $0 \mathrm{~h}$ & $94.17 \pm 6.91$ & $63.67 \pm 6.74^{\mathrm{a}}$ & $127.17 \pm 8.33^{\mathrm{a}, \mathrm{b}}$ & $124.00 \pm 6.45^{\mathrm{a}, \mathrm{b}}$ & $62.50 \pm 3.21^{\mathrm{a}}$ \\
\hline $1 \mathrm{~h}$ & $93.67 \pm 5.47$ & $65.17 \pm 3.49^{a}$ & $69.83 \pm 5.67^{\mathrm{a}}$ & $77.33 \pm 9.24^{\mathrm{a}, \mathrm{b}}$ & $78.83 \pm 8.42^{\mathrm{a}, \mathrm{b}}$ \\
\hline $2 \mathrm{~h}$ & $95.50 \pm 4.51$ & $66.17 \pm 7.47^{\mathrm{a}}$ & $67.17 \pm 3.66^{\mathrm{a}}$ & $70.33 \pm 8.36^{\mathrm{a}}$ & $82.33 \pm 6.28^{\mathrm{a}, \mathrm{b}}$ \\
\hline $4 \mathrm{~h}$ & $95.17 \pm 3.60$ & $68.50 \pm 4.09^{\mathrm{a}}$ & $69.83 \pm 5.88^{\mathrm{a}}$ & $71.17 \pm 7.73^{\mathrm{a}}$ & $86.83 \pm 8.18^{\mathrm{a}, \mathrm{b}}$ \\
\hline $6 \mathrm{~h}$ & $95.67 \pm 8.24$ & $70.00 \pm 7.77^{\mathrm{a}}$ & $72.50 \pm 5.39^{\mathrm{a}}$ & $72.33 \pm 3.98^{\mathrm{a}}$ & $90.33 \pm 6.53^{\mathrm{b}, \mathrm{c}}$ \\
\hline \multicolumn{6}{|c|}{ LVSP, mmHg } \\
\hline Baseline & $124.17 \pm 14.61$ & $122.83 \pm 9.37$ & $125.50 \pm 11.53$ & $127.67 \pm 8.38$ & $122.83 \pm 12.61$ \\
\hline $0 \mathrm{~h}$ & $125.00 \pm 13.25$ & $93.83 \pm 6.37^{\mathrm{a}}$ & $103.50 \pm 13.13^{\mathrm{a}}$ & $104.50 \pm 9.46^{a}$ & $96.67 \pm 13.46^{\mathrm{a}}$ \\
\hline $1 \mathrm{~h}$ & $127.17 \pm 7.65$ & $84.83 \pm 5.38^{\mathrm{a}}$ & $85.17 \pm 15.94^{\mathrm{a}}$ & $91.17 \pm 9.45^{\mathrm{a}}$ & $93.50 \pm 11.78^{\mathrm{a}}$ \\
\hline $2 \mathrm{~h}$ & $128.17 \pm 4.54$ & $80.33 \pm 4.37^{\mathrm{a}}$ & $81.67 \pm 13.57^{\mathrm{a}}$ & $94.33 \pm 10.03^{\mathrm{a}, \mathrm{b}}$ & $100.67 \pm 10.58^{\mathrm{a}-\mathrm{c}}$ \\
\hline $4 \mathrm{~h}$ & $128.67 \pm 8.66$ & $74.67 \pm 3.98^{a}$ & $73.83 \pm 12.80^{\mathrm{a}}$ & $95.50 \pm 12.32^{\mathrm{a}, \mathrm{b}}$ & $107.33 \pm 11.04^{\mathrm{a}-\mathrm{c}}$ \\
\hline $6 \mathrm{~h}$ & $126.10 \pm 10.51$ & $69.50 \pm 5.24^{\mathrm{a}}$ & $67.83 \pm 15.92^{\mathrm{a}}$ & $99.00 \pm 13.43^{\mathrm{a}, \mathrm{b}}$ & $107.67 \pm 8.89^{\mathrm{a}-\mathrm{c}}$ \\
\hline \multicolumn{6}{|c|}{ LVEDP, mmHg } \\
\hline Baseline & $5.30 \pm 0.68$ & $5.12 \pm 0.76$ & $5.14 \pm 0.69$ & $5.12 \pm 0.71$ & $5.17 \pm 0.85$ \\
\hline $0 \mathrm{~h}$ & $5.29 \pm 0.85$ & $6.21 \pm 0.62$ & $6.20 \pm 0.69$ & $6.26 \pm 0.40$ & $6.20 \pm 0.59$ \\
\hline $1 \mathrm{~h}$ & $5.26 \pm 0.68$ & $6.56 \pm 0.83^{\mathrm{a}}$ & $6.89 \pm 0.86^{\mathrm{a}}$ & $6.65 \pm 0.61^{\mathrm{a}}$ & $6.37 \pm 0.56^{\mathrm{a}}$ \\
\hline $2 \mathrm{~h}$ & $5.24 \pm 0.63$ & $7.23 \pm 0.81^{\mathrm{a}}$ & $7.59 \pm 0.65^{\mathrm{a}}$ & $7.21 \pm 0.66^{\mathrm{a}}$ & $6.61 \pm 0.54^{\mathrm{a}}$ \\
\hline $4 \mathrm{~h}$ & $5.25 \pm 0.62$ & $8.82 \pm 0.67^{\mathrm{a}}$ & $9.31 \pm 1.04^{\mathrm{a}}$ & $8.23 \pm 0.86^{\mathrm{a}}$ & $7.02 \pm 0.50^{\mathrm{a}, \mathrm{c}}$ \\
\hline $6 \mathrm{~h}$ & $5.25 \pm 0.66$ & $10.96 \pm 0.65^{\mathrm{a}}$ & $11.26 \pm 1.11^{\mathrm{a}}$ & $9.30 \pm 0.75^{\mathrm{a}-\mathrm{c}}$ & $7.75 \pm 0.56^{\mathrm{a}-\mathrm{c}}$ \\
\hline \multicolumn{6}{|c|}{$+\mathrm{LVdP} / \mathrm{dt} \max , \mathrm{mmHg} / \mathrm{sec}$} \\
\hline Baseline & $9,064.33 \pm 672.53$ & $9,291.00 \pm 501.27$ & $9,162.17 \pm 337.50$ & $9,200.83 \pm 471.40$ & $9,173.17 \pm 390.54$ \\
\hline $0 \mathrm{~h}$ & $9,169.00 \pm 607.90$ & $4,067.00 \pm 524.70^{\mathrm{a}}$ & $8,088.00 \pm 441.94^{\mathrm{a}, \mathrm{b}}$ & $8,173.50 \pm 456.83^{\mathrm{a}, \mathrm{b}}$ & $4,114.50 \pm 513.62^{\mathrm{a}}$ \\
\hline $1 \mathrm{~h}$ & $9,172.17 \pm 483.60$ & $3,792.17 \pm 520.68^{\mathrm{a}}$ & $4,949.67 \pm 298.39^{\mathrm{a}, \mathrm{b}}$ & $5,831 \cdot 17 \pm 445.76^{\mathrm{a}, \mathrm{b}}$ & $4,643.00 \pm 650.51^{\mathrm{a}, \mathrm{b}}$ \\
\hline $2 \mathrm{~h}$ & $9,183.00 \pm 434.36$ & $3,614.83 \pm 448.41^{\mathrm{a}}$ & $3,888.83 \pm 235.35^{\mathrm{a}}$ & $4,657.50 \pm 314.39^{\mathrm{a}-\mathrm{c}}$ & $5,030.83 \pm 610.52^{a-c}$ \\
\hline $4 \mathrm{~h}$ & $9,080.33 \pm 397.61$ & $3,422.83 \pm 422.84^{\mathrm{a}}$ & $3,352.67 \pm 233.10^{\mathrm{a}}$ & $4,220.83 \pm 349.03^{\mathrm{a}-\mathrm{c}}$ & $5,241.33 \pm 565.12^{\mathrm{a}-\mathrm{c}}$ \\
\hline $6 \mathrm{~h}$ & $9,129.85 \pm 436.42$ & $3,258.33 \pm 381.57^{\mathrm{a}}$ & $2,961.50 \pm 229.66^{\mathrm{a}}$ & $4,227.00 \pm 426.64^{\mathrm{a}-\mathrm{c}}$ & $5,392.67 \pm 574.19^{a-c}$ \\
\hline \multicolumn{6}{|c|}{$-\mathrm{LVdP} / \mathrm{dt} \max , \mathrm{mmHg} / \mathrm{sec}$} \\
\hline Baseline & $6,218.67 \pm 339.28$ & $6,260.17 \pm 313.37$ & $6,098.67 \pm 423.04$ & $6,418.67 \pm 430.05$ & $6,423.00 \pm 422.07$ \\
\hline $0 \mathrm{~h}$ & $6,122.00 \pm 382.32$ & $3,491.33 \pm 470.50^{\mathrm{a}}$ & $4,146.67 \pm 345.36^{\mathrm{a}, \mathrm{b}}$ & $4,205.17 \pm 577.13^{a, b}$ & $3,801.17 \pm 411.00^{\mathrm{a}}$ \\
\hline $1 \mathrm{~h}$ & $6,193.50 \pm 330.42$ & $3,304.00 \pm 377.34^{\mathrm{a}}$ & $3,615.67 \pm 458.70^{\mathrm{a}}$ & $3,687.00 \pm 438.40^{\mathrm{a}}$ & $3,779.00 \pm 585.96^{\mathrm{a}}$ \\
\hline $2 \mathrm{~h}$ & $6,183.00 \pm 434.36$ & $2,991.17 \pm 390.47^{\mathrm{a}}$ & $2,884.33 \pm 413.70^{\mathrm{a}}$ & $3,331.33 \pm 578.78^{\mathrm{a}}$ & $3,480.33 \pm 643.92^{\mathrm{a}}$ \\
\hline $4 \mathrm{~h}$ & $6,161.33 \pm 441.93$ & $2,656.83 \pm 308.36^{\mathrm{a}}$ & $2,412.50 \pm 355.95^{\mathrm{a}}$ & $2,937.50 \pm 598.08^{\mathrm{a}}$ & $3,224.83 \pm 567.76^{\mathrm{a}}$ \\
\hline $6 \mathrm{~h}$ & $6,139.09 \pm 490.82$ & $2,507.50 \pm 407.66^{\mathrm{a}}$ & $2,104.00 \pm 177.22^{\mathrm{a}}$ & $2,630.67 \pm 686.80^{\mathrm{a}}$ & $2,980.67 \pm 628.92^{\mathrm{a}}$ \\
\hline
\end{tabular}

Electrocardiogram was performed at baseline (prior to surgery) and at $0,1,2,4$ and $6 \mathrm{~h}$ following ROSC. $\mathrm{n}=6$ per group at each time-point; however, the same batch of animals were used for 0 and $1 \mathrm{~h}$ following ROSC time-points. Data are presented as the mean \pm standard deviation. ${ }^{\mathrm{a} P}<0.05$ vs. sham group; ${ }^{\text {}} \mathrm{P}<0.05$ vs. vehicle group; ${ }^{\mathrm{C}} \mathrm{P}<0.05$ vs. EP group. EP, epinephrine; EPO, erythropoietin; HR, heart rate; MAP, mean arterial pressure; LVSP, left ventricular systolic pressure; LVEDP, left ventricular end-diastolic pressure; +LVdP/dt max, maximal ascending rate of left ventricular pressure during left ventricular isovolumic contraction; -LVdP/dt max, maximal descending rate of left ventricular pressure during left ventricular isovolumic relaxation; ROSC, return of spontaneous circulation. 
Table IV. Alterations in the serum levels of renin in each group.

\begin{tabular}{lccccc}
\hline & \multicolumn{3}{c}{ Group } \\
\cline { 2 - 6 } Time-point & Sham & Vehicle & EP & EPO & EPO + EP \\
\hline Baseline & $2.64 \pm 0.22$ & $2.66 \pm 0.26$ & $2.23 \pm 0.48$ & $2.54 \pm 0.32$ & $51 \pm 0.28$ \\
$2 \mathrm{~h}$ & $2.62 \pm 0.25$ & $56.77 \pm 6.12^{\mathrm{a}}$ & $81.67 \pm 9.85^{\mathrm{a}, \mathrm{b}}$ & $52.42 \pm 6.50^{\mathrm{a}, \mathrm{c}}$ & $59.62 \pm 6.66^{\mathrm{a}, \mathrm{c}}$ \\
$4 \mathrm{~h}$ & $2.67 \pm 0.23$ & $53.63 \pm 4.32^{\mathrm{a}}$ & $73.90 \pm 5.65^{\mathrm{a}, \mathrm{b}}$ & $46.10 \pm 5.96^{\mathrm{a}, \mathrm{c}}$ & $59.77 \pm 10.91^{\mathrm{a}, \mathrm{c}}$ \\
$6 \mathrm{~h}$ & $2.91 \pm 0.31$ & $46.25 \pm 2.94^{\mathrm{a}}$ & $58.97 \pm 5.07^{\mathrm{a}, \mathrm{b}}$ & $31.81 \pm 5.00^{\mathrm{a}, \mathrm{c}}$ & $52.76 \pm 3.34^{\mathrm{a}}$ \\
\hline
\end{tabular}

Renin levels are presented as $\mathrm{ng} / \mathrm{ml}$ and were measured in the serum at baseline (prior to surgery) and at 2,4 and $6 \mathrm{~h}$ following return of spontaneous circulation. Data are presented as the mean \pm standard deviation, $\mathrm{n}=6 /$ group per time-point. ${ }^{\mathrm{a}} \mathrm{P}<0.01 \mathrm{vs}$. sham group; ${ }^{\mathrm{b}}<<0.01$ vs. vehicle group; ${ }^{\mathrm{C}}<0.01$ vs. EP group. EP, epinephrine; EPO, erythropoietin.

Table V. Alterations in the serum levels of Ang II in each group.

\begin{tabular}{|c|c|c|c|c|c|}
\hline \multirow[b]{2}{*}{ Time-point } & \multicolumn{5}{|c|}{ Group } \\
\hline & Sham & Vehicle & EP & $\mathrm{EPO}+\mathrm{EP}$ & EPO \\
\hline Baseline & $6.72 \pm 2.43$ & $6.68 \pm 2.22$ & $6.38 \pm 3.88$ & $6.73 \pm 2.35$ & $6.69 \pm 3.22$ \\
\hline $2 \mathrm{~h}$ & $6.88 \pm 2.25$ & $10.13 \pm 3.56^{\mathrm{a}}$ & $20.18 \pm 4.94^{\mathrm{a}, \mathrm{b}}$ & $14.61 \pm 2.14^{\mathrm{a}, \mathrm{b}}$ & $10.16 \pm 2.82^{\mathrm{a}, \mathrm{c}}$ \\
\hline $4 \mathrm{~h}$ & $6.32 \pm 2.42$ & $10.12 \pm 5.47^{\mathrm{a}}$ & $18.25 \pm 6.10^{\mathrm{a}, \mathrm{b}}$ & $18.03 \pm 4.09^{\mathrm{a}, \mathrm{b}}$ & $8.99 \pm 1.39^{\mathrm{a}, \mathrm{c}}$ \\
\hline $6 \mathrm{~h}$ & $5.93 \pm 2.33$ & $10.09 \pm 4.00^{\mathrm{a}}$ & $16.42 \pm 4.62^{\mathrm{a}, \mathrm{b}}$ & $15.25 \pm 4.71^{\mathrm{a}, \mathrm{b}}$ & $8.23 \pm 3.15^{\mathrm{a}, \mathrm{c}}$ \\
\hline
\end{tabular}

Ang II levels are presented as $\mathrm{ng} / \mathrm{ml}$ and were measured in the serum at baseline (prior to surgery) and at 2, 4 and $6 \mathrm{~h}$ following return of spontaneous circulation. Data are presented as the mean \pm standard deviation $(\mathrm{ng} / \mathrm{ml}) . \mathrm{n}=6 /$ group per time-point. ${ }^{\mathrm{a}} \mathrm{P}<0.01 \mathrm{vs}$. sham group ${ }^{\mathrm{b}} \mathrm{P}<0.01$ vs. vehicle group.; ${ }^{\mathrm{C}}<0.01$ vs. EP group. Ang II, angiotensin II; EP, epinephrine; EPO, erythropoietin.

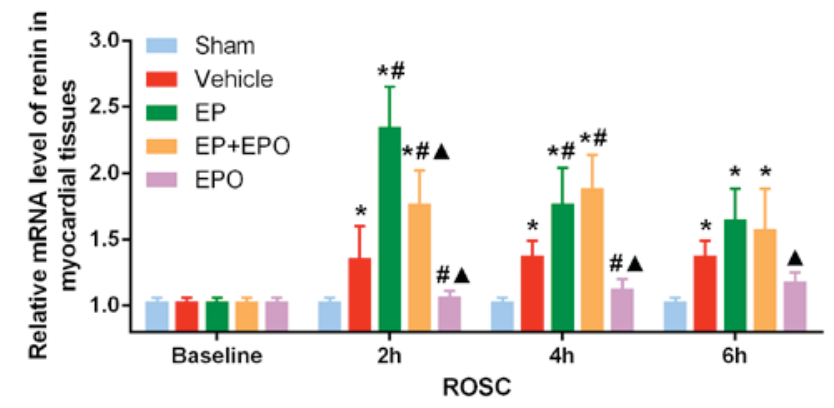

Figure 2. Alterations in the mRNA level of renin in myocardial tissues. Sprague-Dawley rats were randomly divided into five groups with $n=30$ per group: Sham-operated group, CA resuscitation group, CA resuscitation $+\mathrm{EP}$ group, $\mathrm{CA}$ resuscitation $+\mathrm{EPO}$ group and $\mathrm{CA}$ resuscitation $+\mathrm{EP}+\mathrm{EPO}$ group. Myocardial tissues were obtained from the five groups at baseline (prior to surgery) and at 2, 4 and $6 \mathrm{~h}$ after ROSC, with $\mathrm{n}=6$ per group at each time-point. The mRNA level of renin in myocardial tissues was measured by reverse transcription-quantitative polymerase chain reaction. Comparisons among multiple subgroups were performed using two-way analysis of variance. ${ }^{*} \mathrm{P}<0.05$ vs. sham group; ${ }^{\#} \mathrm{P}<0.05$ vs. vehicle group; ${ }^{\wedge} \mathrm{P}<0.05$ vs. EP group. CA, cardiac arrest; EP, epinephrine; EPO, erythropoietin; ROSC, return of spontaneous circulation; Sham group, sham-operated; vehicle group, CA resuscitation; EP group, CA resuscitation + EP; EPO group, CA resuscitation + EPO; EP + EPO group, CA resuscitation + EP + EPO.

to the protein expression of AT1R (Fig. 3B), were increased in myocardial tissues of the vehicle group (all $\mathrm{P}<0.05$ ), indicating that $\mathrm{CA}$ resuscitation may activate the canonical RAS signaling of renin-AngII-AT1R.
The mRNA levels of AT1R were not altered in the EPO group compared with the vehicle group (Fig. 3A). EPO suppressed the increase of AT1R protein in myocardial tissues to an extent at $2 \mathrm{~h}$ after ROSC $(\mathrm{P}<0.05$, EPO group vs. vehicle group; Fig. 3B), however, the regulation was not maintained between 4 and $6 \mathrm{~h}$ after ROSC $(\mathrm{P}>0.05$, EPO group vs. vehicle group; Fig. 3B). The myocardial expression of AT1R mRNA was reduced in the EP + EPO group compared with the EP group at $2 \mathrm{~h}$ following $\operatorname{ROSC}(\mathrm{P}<0.05$; Fig. 3A), but the difference disappeared later (both $\mathrm{P}>0.05$, EP+EPO group vs. EP group, 4 and $6 \mathrm{~h}$ after ROSC; Fig. 3A). The myocardial expression of AT1R protein was reduced in the EP + EPO group compared with the EP group at 4 and $6 \mathrm{~h}$ following ROSC $(\mathrm{P}<0.05$; Fig. 3B), although the levels in the EP + EPO group remained significantly higher compared with the vehicle group $(\mathrm{P}<0.05$; Fig. $3 \mathrm{~B})$. EP addition further increased the myocardial expression of renin and AT1R (both $\mathrm{P}<0.05$, EP group vs. vehicle group; Figs. 2 and 3).

Overall, the results indicated that the effects of EPO in alleviating post-resuscitation myocardial dysfunction (Table III) may be mediated through mechanisms other than direct inhibition of the renin-Ang II-AT1R signaling pathway.

EPO enhances AT2R expression in myocardial tissues post-resuscitation. The myocardial expression of AT2R 

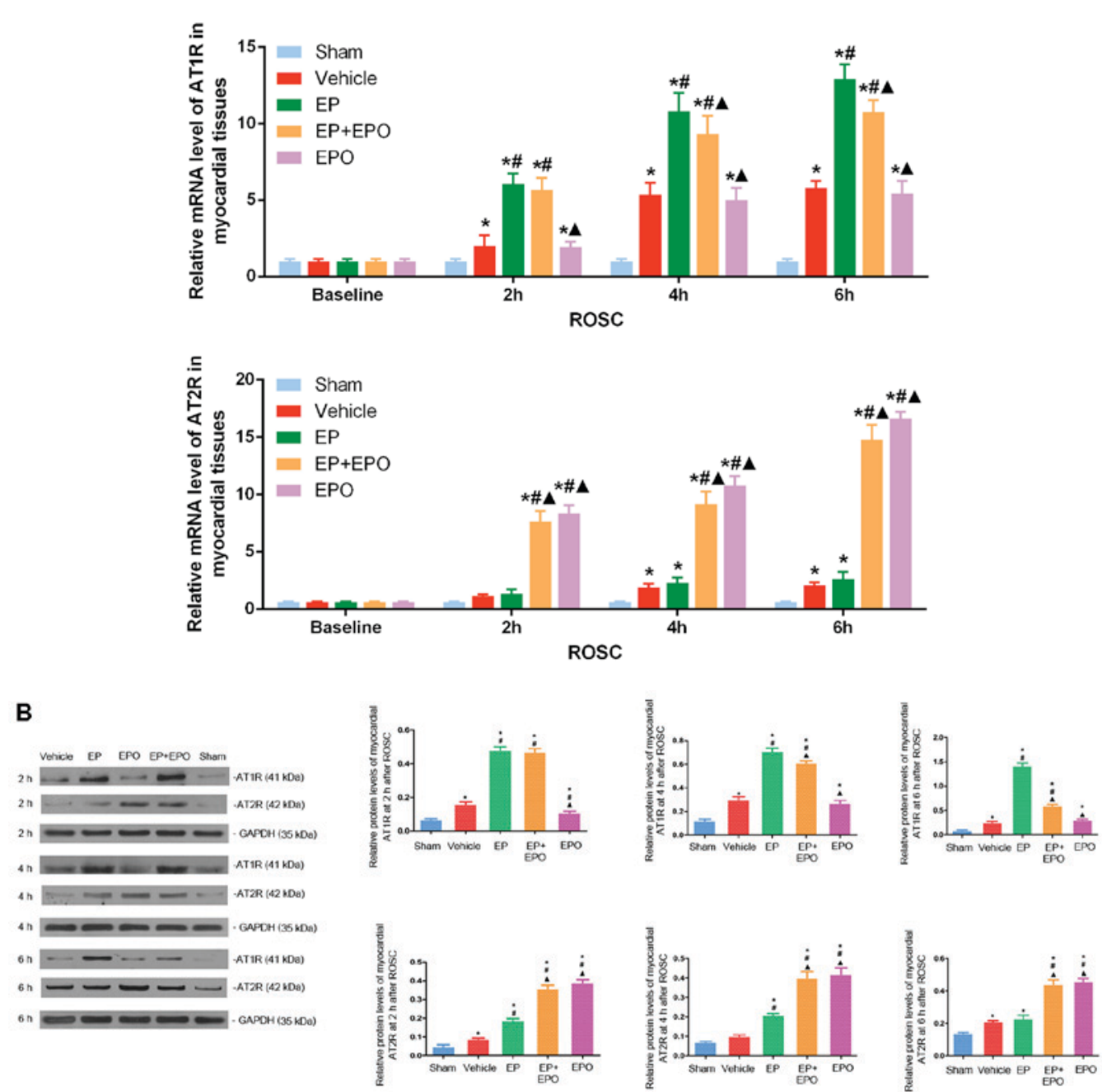

Figure 3. Alterations in the mRNA and protein levels of AT1R and AT2R in myocardial tissues. Sprague-Dawley rats were randomly divided into five groups with $\mathrm{n}=30$ per group: Sham-operated group, CA resuscitation group, CA resuscitation + EP group, CA resuscitation + EPO group and CA resuscitation + EP + EPO group. Myocardial tissues were obtained from the five groups at baseline (prior to surgery) and at 2, 4 and $6 \mathrm{~h}$ after ROSC, with $\mathrm{n}=6 \mathrm{per}$ group at each time-point. (A) mRNA levels of AT1R and AT2R in myocardial tissues at different time-points. (B) Protein expressions of AT1R and AT2R in myocardial tissues at different time-points. Representative western blot bands are presented. Comparisons among multiple subgroups were performed using two-way analysis of variance. ${ }^{\mathrm{P}}<0.05$ vs. sham group; ${ }^{\mathrm{P}} \mathrm{P}<0.05$ vs. vehicle group; ${ }^{\mathbf{}} \mathrm{P}<0.05$ vs. EP group. AT1R, angiotensin II receptor type 1 ; AT2R, angiotensin II receptor type 2; CA, cardiac arrest; EP, epinephrine; EPO, erythropoietin; ROSC, return of spontaneous circulation; Sham group, sham-operated; vehicle group, CA resuscitation; EP group, CA resuscitation + EP; EPO group, CA resuscitation + EPO; EP + EPO group, CA resuscitation + EP + EPO

mRNA and protein was increased upon $\mathrm{CA}$ resuscitation $(\mathrm{P}<0.05$, vehicle group vs. sham group; Fig. 3). The addition of EP enhanced this increase in AT2R expression significantly at the protein level at 2 and $4 \mathrm{~h}$ after $\operatorname{ROSC}(\mathrm{P}<0.05$, EP group vs. vehicle group; Fig. 3B). Furthermore, EPO pretreatment in the EPO and EP + EPO groups led to even higher myocardial AT2R mRNA and protein levels $(\mathrm{P}<0.05$, EPO group vs. EP and vehicle groups, and EP + EPO group vs. EP and vehicle groups; Fig. 3).

Taken together, the results indicate that the activated renin-AngII-AT1R signaling upon CA resuscitation may contribute to myocardial dysfunction.

Based on the results of the present study, it may be preliminarily inferred that the mechanisms underlying the effects of EPO in alleviating post-resuscitation myocardial dysfunction may be associated with the increase of AT2R expression at mRNA and protein levels, which may counteract the canonical signaling mediated by AT1R.

\section{Discussion}

Following CA, the interruption of blood flow and reperfusion injury following resuscitation may lead to intractable low blood pressure and recurrent malignant ventricular arrhythmia, causing complex pathophysiological alterations in major organs. This condition is termed post-resuscitation syndrome and has an incidence of 30-70\% in heart disease, and is also one of the most important reasons for early mortality following resuscitation $(19,20)$.

The RAS is thought to have an important role in post-resuscitation syndrome. This system regulates blood pressure and consists of endocrine, autocrine and paracrine factors $(8,21)$. RAS activation, both systemic and local, is an important step in chronic myocardial remodeling and one of the decisive factors in the prognosis of myocardial infarction (22). Furthermore, clinical trials have demonstrated that RAS inhibitors reduced the 30-day all-cause rehospitalization 
rate in patients that suffered heart failure (23). In RAS, Ang I is converted into Ang II by angiotensin-converting enzyme (ACE). When Ang II binds to AT1R in the myocardium, the Ang II-AT1R axis acts as a detrimental effector, causing myocardial cell damage and development of heart failure in the following manner: Development of myocardial hypertrophy and increased production of reactive oxygen free radicals leads to cell mitochondrial damage and myocardial cell apoptosis $(21,24)$; promotion of the expression of proinflammatory transcription factors, nuclear factor- $\kappa \mathrm{B}$ and interleukin- 6 in cells, which subsequently promotes inflammatory reactions; through the action of NADPH oxidase, ultimately increasing the production of reactive oxygen free radicals, which causes damage to cell mitochondria, myocardial hypertrophy, and myocardial fibrosis and dysfunction (25); and through the phosphatidylinositol 3-kinase/Akt pathway, wherein Akt phosphorylation is reduced and cardiac hypertrophy and cardiomyocyte autophagy are promoted, leading to heart failure (26). Strohmenger et al (27) demonstrated that administration of the Ang II antagonist telmisartan during the port-resuscitation phase in pigs improved myocardial contractility. Wang et al (4) also reported that following CA, the use of sildenafil inhibited the ACE-Ang II-AT1R axis, which reduced myocardial ischemia-reperfusion injury. In addition, Kaschina et al (28) revealed that indirect stimulation of AT2R reduced myocardial hypertrophy and fibrosis following myocardial infarction, thereby improving cardiac function, however, this was accompanied by hypotension. Furthermore, $\mathrm{Xu}$ et al (29) demonstrated that high AT2R expression in myocardial cells inhibited myocardial oxidative stress, reduced myocardial hypertrophy and myocardial fibrosis, inhibited ventricular remodeling, and improved cardiac function. Taken together, the results of these studies indicated that counteracting the Ang II-AT1R axis may have an important role in ameliorating post-resuscitation myocardial dysfunction, as supported by an additional previous study (30).

In the present study, lower MAP, LVSP, $+\mathrm{LVdP} / \mathrm{dt}$ max and $-\mathrm{LVdP} / \mathrm{dt}$ max, and higher LVEDP, were observed in rats post-resuscitation, compared with the sham group (Table III). The serum levels of renin and Ang II were elevated upon CA resuscitation, as was the myocardial expression of renin and AT1R, compared with the sham group. These results indicated that CPR following CA may activate renin-Ang II-AT1R signaling, which may contribute to myocardial dysfunction, consistent with previous studies $(4,26,28)$.

EPO has been reported to exert protective effects on various tissues and organs (31). The role of EPO in cardiac function protection and reducing post-resuscitation myocardial dysfunction is well-established $(5,9,11,14,32,33)$; however, to the best of our knowledge, its modulation of the RAS has not been previously investigated. Given the established roles of the RAS in myocardial ischemia-reperfusion injury following $\mathrm{CA}$, it may be hypothesized that EPO may alleviate post-resuscitation myocardial dysfunction by regulating the RAS. The results of the present study indicated that EPO alleviated post-resuscitation myocardial dysfunction, as higher MAP, LVSP and +LVdP/dt max, and lower LVEDP, was observed following ROSC in the EP group, compared with the vehicle group, which was consistent with previous findings $(32,33)$.
Within the RAS, EPO does not only mediate AT1R, but also has effects on AT2R, nitric oxide levels, NAPDH oxidase 4 and heme oxygenase-1, in addition to other complex signaling pathways. Patients with congenital AT1R resistance were reported to exhibit increased EPO secretion (34). The present study demonstrated that the most noticeable effect of EPO treatment was the upregulation of AT2R expression in myocardial tissues, rather than the downregulation of myocardial AT1R or suppression of renin and Ang II levels, which were not significant in most cases, with regard to its regulation of RAS upon CA resuscitation. Therefore, these results indicate that the effects of EPO in alleviating post-resuscitation myocardial dysfunction may primarily rely on AT2R activation, which counteracts the canonical signaling mediated by AT1R. However, AT2R phosphorylation requires investigation in future studies to reach a firm conclusion concerning AT2R activation by EPO following CA. In addition, mechanistic studies using antagonists or knockdown strategies are required to confirm whether AT2R mediates the effects of EPO following CA resuscitation. Furthermore, as high AT2R expression in myocardial cells has been reported to inhibit oxidative stress (29), the measurement of oxidative stress markers in myocardial tissues is also required in future studies. Finally, it should be noted that the long-term effects of EPO were somewhat compromised when combined with EP addition (Table III), however the myocardial expression of AT2R protein was almost identical between the EPO and EP + EPO groups, which indicates that, in addition to enhancing the AT2R expression, additional mechanisms may be involved in mediating the effects of EPO upon CA resuscitation, which should also be investigated.

$\mathrm{EP}$, which is the resuscitation drug that is currently recommended in the guidelines, exerts strong $\beta$ - and $\alpha$-adrenergic activation effects (35), which may aggravate myocardial ischemic injury (36). Therefore, the present study was designed to include a group with EP addition as EP may be involved in the mechanisms underlying post-resuscitation myocardial dysfunction. However, according to the results of the present study, EP addition did not exacerbate the myocardial dysfunction post-resuscitation, although it did increase the production/expression of renin, Ang II and AT1R. One potential explanation for this discrepancy is that the maximum effect of renin-Ang II-AT1R signaling is already reached upon CA resuscitation alone. In addition, the increase in AT2R protein expression induced by EP addition at 2 and $4 \mathrm{~h}$ after ROSC may also contribute to the counteractive regulation. However, these hypotheses require further investigation.

The primary limitation of the present study is that study planning did not include a sham + EPO group. Such a group would clarify the effect of EPO alone on myocardial dysfunction upon CA resuscitation. Additional confirmation of EPO effects with this control group should be performed in future studies.

In conclusion, the results of the current study indicate that EPO may ameliorate the myocardial dysfunction upon CA resuscitation, and the underlying mechanisms may include counteracting the canonical AT1R-mediated RAS signaling as a result of enhanced AT2R expression, rather than direct inhibition of the renin-Ang II-AT1R signaling pathway. Further investigation is required to determine these mechanisms in detail. 


\section{Acknowledgements}

The present study was supported by the Project of the Department of Science and Technology, Guizhou province. [grant nos. (2012)014 and (2016)1411].

\section{References}

1. Jentzer JC, Chonde MD and Dezfulian C: Myocardial dysfunction and shock after cardiac arrest. Biomed Res Int 2015: 314796, 2015.

2. Bougouin W and Cariou A: Management of postcardiac arrest myocardial dysfunction. Curr Opin Crit Care 19: 195-201, 2013.

3. Rivers EP, Wortsman J, Rady MY, Blake HC, McGeorge FT and Buderer NM: The effect of the total cumulative epinephrine dose administered during human CPR on hemodynamic, oxygen transport, and utilization variables in the postresuscitation period. Chest 106: 1499-1507, 1994.

4. Wang G, Zhang Q, Yuan W, Wu J and Li C: Sildenafil protects against myocardial ischemia-reperfusion injury following cardiac arrest in a porcine model: Possible role of the renin-angiotensin system. Int J Mol Sci 16: 27015-27031, 2015.

5. Huang CH, Hsu CY, Chen HW, Tsai MS, Cheng HJ, Chang CH, Lee YT and Chen WJ: Erythropoietin improves the postresuscitation myocardial dysfunction and survival in the asphyxia-induced cardiac arrest model. Shock 28: 53-58, 2007.

6. Mollace V, Gliozzi M, Capuano A and Rossi F: Modulation of RAAS-natriuretic peptides in the treatment of HF: Old guys and newcomers. Int J Cardiol 226: 126-131, 2017.

7. Inuzuka T, Fujioka Y, Tsuda M, Fujioka M, Satoh AO, Horiuchi K, Nishide S, Nanbo A, Tanaka S and Ohba Y: Attenuation of ligand-induced activation of angiotensin II type 1 receptor signaling by the type 2 receptor via protein kinase C. Sci Rep 6: 21613, 2016.

8. KaschinaE,Grzesiak A,Li J,Foryst-Ludwig A,TimmM,Rompe F, Sommerfeld M, Kemnitz UR, Curato C, Namsolleck P, et al: Angiotensin II type 2 receptor stimulation: A novel option of therapeutic interference with the renin-angiotensin system in myocardial infarction? Circulation 118: 2523-2532, 2008.

9. Borovnik-Lesjak V, Whitehouse K, Baetiong A, Artin B, Radhakrishnan J and Gazmuri RJ: High-dose erythropoietin during cardiac resuscitation lessens postresuscitation myocardial stunning in swine. Transl Res 162: 110-121, 2013.

10. Nandra KK, Collino M, Rogazzo M, Fantozzi R, Patel NS and Thiemermann C: Pharmacological preconditioning with erythropoietin attenuates the organ injury and dysfunction induced in a rat model of hemorrhagic shock. Dis Model Mech 6: 701-709, 2013.

11. Sanchis-Gomar F, Garcia-Gimenez JL, Pareja-Galeano H, Romagnoli M, Perez-Quilis C and Lippi G: Erythropoietin and the heart: Physiological effects and the therapeutic perspective. Int J Cardiol 171: 116-125, 2014

12. Jie KE, Verhaar MC, Cramer MJ, van der Putten K, Gaillard CA, Doevendans PA, Koomans HA, Joles JA and Braam B: Erythropoietin and the cardiorenal syndrome: Cellular mechanisms on the cardiorenal connectors. Am J Physiol Renal Physiol 291: F932-F944, 2006.

13. Rosario R and Epstein M: Relationship between erythropoietin administration and alterations of renin-angiotensin-aldosterone. J Renin Angiotensin Aldosterone Syst 7: 135-138, 2006.

14. Lundby C, Thomsen JJ, Boushel R, Koskolou M, Warberg J, Calbet JA and Robach P: Erythropoietin treatment elevates haemoglobin concentration by increasing red cell volume and depressing plasma volume. J Physiol 578: 309-314, 2007.

15. Cho A and Seok SH: Ethical guidelines for use of experimental animals in biomedical research. J Bacteriol Virol 43: 18-26, 2013.

16. de Prost N, Ricard JD, Saumon G and Dreyfuss D: Ventilator-induced lung injury: Historical perspectives and clinical implications. Ann Intensive Care 1: 28, 2011.

17. Wang P, Yao L, Zhou LL, Liu YS, Chen MD, Wu HD, Chang RM, Li Y, Zhou MG, Fang XS, et al: Carbon monoxide improves neurologic outcomes by mitochondrial biogenesis after global cerebral ischemia induced by cardiac arrest in rats. Int J Biol Sci 12: 1000-1009, 2016.

18. Livak KJ and Schmittgen TD: Analysis of relative gene expression data using real-time quantitative PCR and the 2(-Delta Delta C(T)) method. Methods 25: 402-408, 2001
19. Mongardon N, Dumas F, Ricome S, Grimaldi D, Hissem T, Pène F and Cariou A: Postcardiac arrest syndrome: From immediate resuscitation to long-term outcome. Ann Intensive Care 1: 45, 2011.

20. Lemiale V, Dumas F, Mongardon N, Giovanetti O, Charpentier J, Chiche JD, Carli P, Mira JP, Nolan J and Cariou A: Intensive care unit mortality after cardiac arrest: The relative contribution of shock and brain injury in a large cohort. Intensive Care Med 39: 1972-1980, 2013.

21. Zablocki D and Sadoshima J: Angiotensin II and oxidative stress in the failing heart. Antioxid Redox Signal 19: 1095-1109, 2013.

22. Zreikat HH, Harpe SE, Slattum PW, Mays DP, Essah PA and Cheang KI: Effect of Renin-Angiotensin system inhibition on cardiovascular events in older hypertensive patients with metabolic syndrome. Metabolism 63: 392-399, 2014.

23. Sanam K, Bhatia V, Bajaj NS, Gaba S, Morgan CJ, Fonarow GC, Butler J, Deedwania P, Prabhu SD, Wu WC, et al: Renin-Angiotensin system inhibition and lower 30-day all-cause readmission in medicare beneficiaries with heart failure. Am J Med 129: 1067-1073, 2016.

24. Wang X, Yuan B, Dong W, Yang B, Yang Y, Lin X and Gong G: Humid heat exposure induced oxidative stress and apoptosis in cardiomyocytes through the angiotensin II signaling pathway. Heart Vessels 30: 396-405, 2015 .

25. Zhang YH: Neuronal nitric oxide synthase in hypertension - an update. Clin Hypertens 22: 20, 2016.

26. Lin L, Liu X, Xu J, Weng L, Ren J, Ge J and Zou Y: High-density lipoprotein inhibits mechanical stress-induced cardiomyocyte autophagy and cardiac hypertrophy through angiotensin II type 1 receptor-mediated PI3K/Akt pathway. J Cell Mol Med 19: 1929-1938, 2015.

27. Strohmenger HU, Lindner KH, Wienen W and Vogt J: Effects of the AT1-selective angiotensin II antagonist, telmisartan, on hemodynamics and ventricular function after cardiopulmonary resuscitation in pigs. Resuscitation 35: 61-68, 1997.

28. Kaschina E, Lauer D, Schmerler P, Unger T and Steckelings UM: AT2 receptors targeting cardiac protection post-myocardial infarction. Curr Hypertens Rep 16: 441, 2014

29. Xu J, Sun Y, Carretero OA, Zhu L, Harding P, Shesely EG, Dai X, Rhaleb NE, Peterson E and Yang XP: Effects of cardiac overexpression of the angiotensin II type 2 receptor on remodeling and dysfunction in mice post-myocardial infarction. Hypertension 63 : 1251-1259, 2014.

30. Xiao HL, Li CS, Zhao LX, Yang J, Tong N, An L and Liu QT: Captopril improves postresuscitation hemodynamics protective against pulmonary embolism by activating the ACE2/Ang(1-7)/Mas axis. Naunyn Schmiedebergs Arch Pharmacol 389: 1159-1169, 2016

31. Sutherland BA, Minnerup J, Balami JS, Arba F, Buchan AM and Kleinschnitz C: Neuroprotection for ischaemic stroke: Translation from the bench to the bedside. Int J Stroke 7: 407-418, 2012.

32. Ahmet I, Tae HJ, Juhaszova M, Riordon DR, Boheler KR, Sollott SJ, Brines M, Cerami A, Lakatta EG and Talan MI: A small nonerythropoietic helix B surface peptide based upon erythropoietin structure is cardioprotective against ischemic myocardial damage. Mol Med 17: 194-200, 2011.

33. Najjar SS, Rao SV, Melloni C, Raman SV, Povsic TJ, Melton L, Barsness GW, Prather K, Heitner JF, Kilaru R, et al: Intravenous erythropoietin in patients with ST-segment elevation myocardial infarction: REVEAL: A randomized controlled trial. JAMA 305: 1863-1872, 2011.

34. Calò LA, Davis PA, Maiolino G, Pagnin E, Ravarotto V, Naso E, Carraro G and Naso A: Assessing the relationship of angiotensin II Type 1 receptors with erythropoietin in a human model of endogenous angiotensin II Type 1 receptor antagonism. Cardiorenal Med 6: 16-24, 2015.

35. Ahles A and Engelhardt S: Polymorphic variants of adrenoceptors: Pharmacology, physiology, and role in disease. Pharmacol Rev 66: 598-637, 2014.

36. Broadley KJ and Penson PE: The roles of alpha- and beta-adrenoceptor stimulation in myocardial ischaemia. Auton Autacoid Pharmacol 24: 87-93, 2004.

This work is licensed under a Creative Commons Attribution-NonCommercial-NoDerivatives 4.0 International (CC BY-NC-ND 4.0) License. 